\title{
Editorial
}

\section{Some Recent Trends in Variational Inequalities and Optimization Problems with Applications}

\author{
Abdellah Bnouhachem, ${ }^{1,2}$ Abdelouahed Hamdi, ${ }^{3}$ and Xu Minghua ${ }^{4}$ \\ ${ }^{1}$ School of Management Science and Engineering, Nanjing University, Nanjing 210093, China \\ ${ }^{2}$ Ibn Zohr University, ENSA, BP 1136, Agadir, Morocco \\ ${ }^{3}$ Department of Mathematics, Statistics and Physics, College of Arts and Sciences, Qatar University, PB 2713, Doha, Qatar \\ ${ }^{4}$ School of Mathematics and Physics, Changzhou University, Changzhou, Jiangsu Province 213164, China
}

Correspondence should be addressed to Abdellah Bnouhachem; babedallah@yahoo.com

Received 26 November 2013; Accepted 26 November 2013; Published 23 January 2014

Copyright (C) 2014 Abdellah Bnouhachem et al. This is an open access article distributed under the Creative Commons Attribution License, which permits unrestricted use, distribution, and reproduction in any medium, provided the original work is properly cited.

Variational inequalities theory, which was introduced in the sixties, has emerged as an interesting and fascinating branch of applicable mathematics with a wide range of applications in industry, finance, economics, social, and pure and applied sciences. This field is dynamic and is experiencing an explosive growth in both theory and applications; as a consequence, research techniques and problems are drawn from various fields. The ideas and techniques of variational inequalities are being applied in a variety of diverse areas of sciences and prove to be productive and innovative. It has been shown that this theory provides the most natural, direct, simple, unified, and efficient framework for a general treatment of a wide class of unrelated linear and nonlinear problems. Variational inequalities have been extended and generalized in several directions using novel and new techniques. In parallel, optimization methods based on proximal point and proximal-like type methods have attracted a large number of researchers in the last three decades. In the same spirit, we can cite, for instance, the alternating direction multipliers method, which is based on the augmented lagrangian algorithm, which itself can be seen as a direct application of the proximal point algorithm to the dual problem of a constrained optimization problem.

The aim of this special issue is to present new approaches and theories for variational inequalities arising in mathematics and applied sciences. This special issue includes 14 highquality peer-reviewed papers that deal with different aspects of variational inequalities. These papers contain some new, novel, and innovative techniques and ideas. We hope that all the papers published in this special issue can motivate and foster further scientific works and development of the research in the area of theory, algorithms, and applications of variational inequalities.

The summaries of the 14 papers in this issue are listed as follows.

The paper of C. Chen et al. considers a class of linearly constrained separable convex programming problems without coupled variables. They weaken some conditions to obtain convergence of the alternating direction method of multipliers and they propose also a relaxed ADMM involving an additional computation of optimal step size and establish its global convergence under mild conditions.

The paper of H. Sun and Y. Wang revisits the global error bound for the generalized nonlinear complementarity problem over a polyhedral cone (GNCP) and sharpens the global error bound for the GNCP under weaker conditions, which improves the existing error bound estimation for the problem.

The paper of M. Ma concerns the design and the convergence analysis of algorithms to split variational inequality and equilibrium problems.

The paper of Y. Wang and C. Wang gives a new modified Ishikawa type iteration algorithm for common fixed points of total asymptotically strict pseudocontractive semigroups. 
Strong and weak convergence are proved under mild conditions. Furthermore, the main results presented in this work extend and improve some recent results.

The paper of X. Fu presents an implementable proximal step by a slight relaxation to the subproblem of proximal point algorithm (PPA) to solve linearly constrained convex programming. Self-adaptive strategies are proposed to improve the convergence in practice. The paper also discusses some applications and performs some numerical experiments to confirm the efficiency of the proposed method.

The paper of $\mathrm{H}$. Xu establishes the strong convergence of prediction-correction and relaxed hybrid steepest-descent method (PRH method) for variational inequalities under some suitable conditions that simplify the proof. Further, the author shows the efficiency of the proposed algorithm through a well-designed set of practical numerical experiments.

The paper of $\mathrm{M}$. Xu et al. considers the study of some matrix optimization problems using the proximal alternating direction method. The authors show that the restriction on the proximal parameters can be relaxed for solving these kinds of problems and give some numerical experiments to conclude that their modified method presents better performance than the classical proximal alternating direction method.

The paper of J.-L. Jiang et al. considers the locations of multiple facilities in the space $\mathbb{R}^{p}$, with the aim of minimizing the sum of weighted distances between facilities and regional customers, where the proximity between a facility and a regional customer is evaluated by the closest distance. And the authors propose a new location-allocation heuristic scheme to solve their problem. Convergence is proved under mild assumptions; and furthermore some preliminary numerical results are reported to show the effectiveness of the new algorithm.

The paper of A. Roldan et al. studies the existence and uniqueness of coincidence point for nonlinear mappings of any number of arguments under a weak $(\psi, \varphi)$-contractivity condition in partial metric spaces. The obtained results generalize, extend, and unify several classical and very recent related results in the literature in metric spaces and in partial metric spaces.

The paper of $Z$. Jia et al. extends the convergence analysis given by Han and Yuan for alternating direction method of multipliers (ADMM) from the strongly convex to a more general case. Further, the authors prove under the assumption that the individual functions are composites of strongly convex functions and linear functions that the classical ADMM for separable convex programming with two blocks can be extended to the case with more than three blocks.

The paper of M. Li and Y. You presents a simple proof for the same convergence rate of the relaxed proximal point algorithm (PPA) in both ergodic and nonergodic senses.

The paper of W.-S. Du et al. extends, generalizes, and improves several fundamental results on the existence (and uniqueness) of coincidence points and fixed points for well-known maps in the literature. Furthermore, some fixed coincidence point theorems for multivalued nonself maps in the context of complete metric spaces are given.

The paper of F. Ma et al. develops, studies, and implements a new prediction-correction method for monotone variational inequalities with separable structure. At each iteration, the proposed algorithm also allows the involved subvariational inequalities to be solved in parallel.

The paper of A. Barbagallo and P. Mauro concerns a dynamic oligopolistic market equilibrium problem in the realistic case in which the presence of capacity constraints and production excesses are allowed and, moreover, the production function depends not only on the time but also on the equilibrium distribution. The authors prove the equivalence between this equilibrium definition and a suitable evolutionary quasi-variational inequality, and they study the analysis of existence, regularity, and sensitivity of solutions.

\section{Acknowledgments}

We would like to express our sincere thanks to the authors for contributing to this issue, as well as to the anonymous reviewers for their generous and accurate refereeing process and their valuable comments and suggestions. Without their evident support this special issue would not have come out. We want to also acknowledge with gratitude the assistance and help provided by the editorial board members of this journal during the preparation of this special issue.

Abdellah Bnouhachem Abdelouahed Hamdi Xu Minghua 


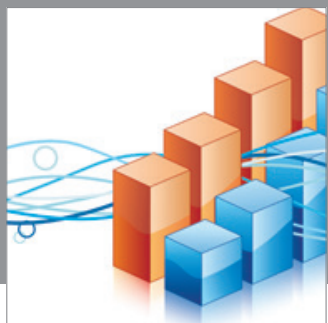

Advances in

Operations Research

mansans

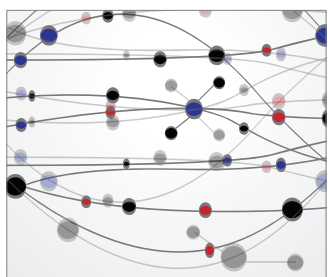

The Scientific World Journal
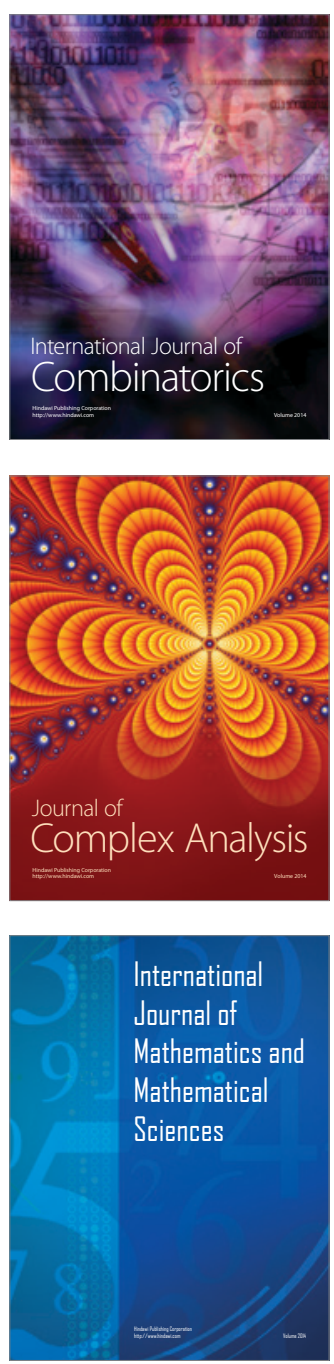
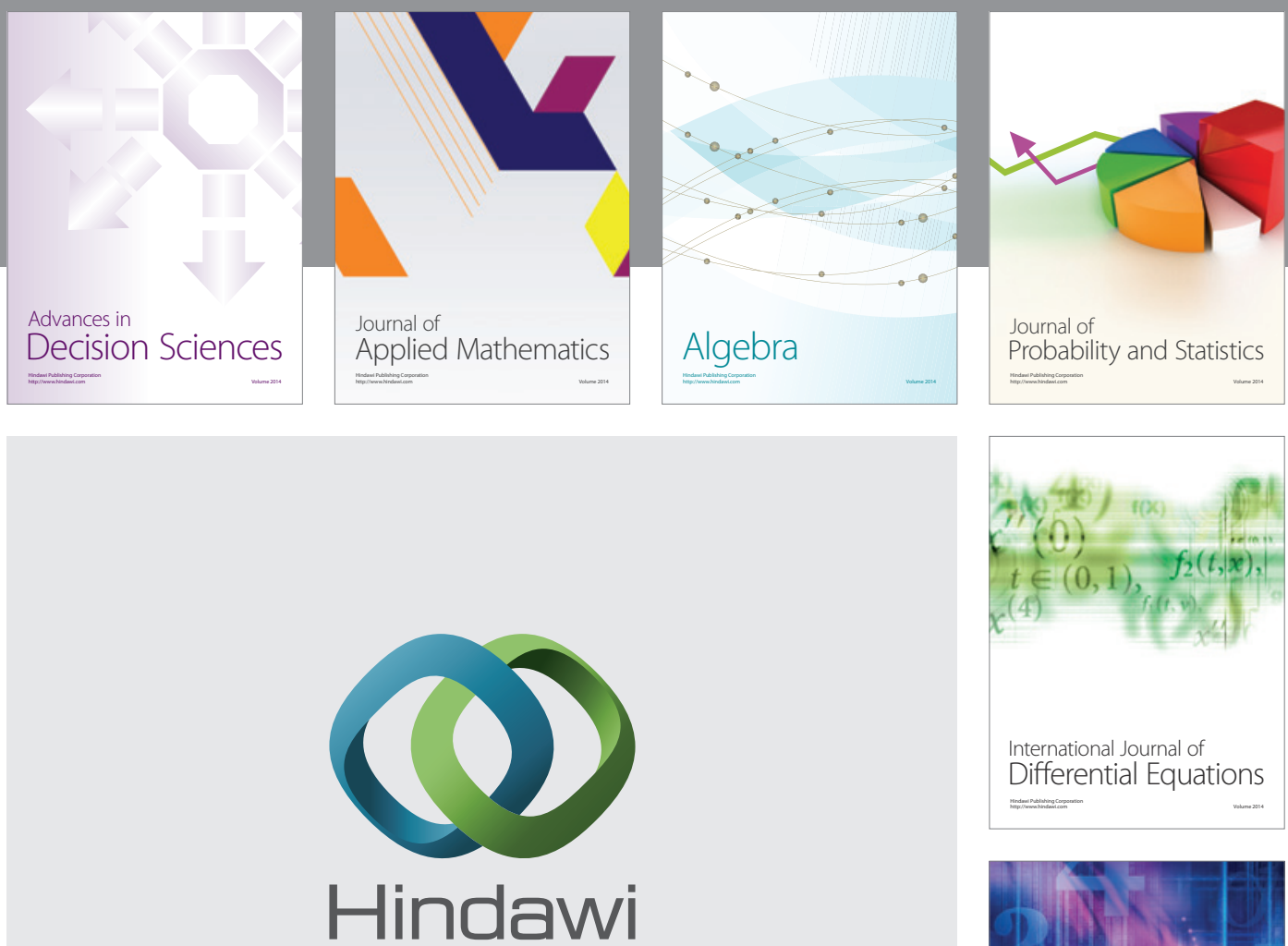

Submit your manuscripts at http://www.hindawi.com
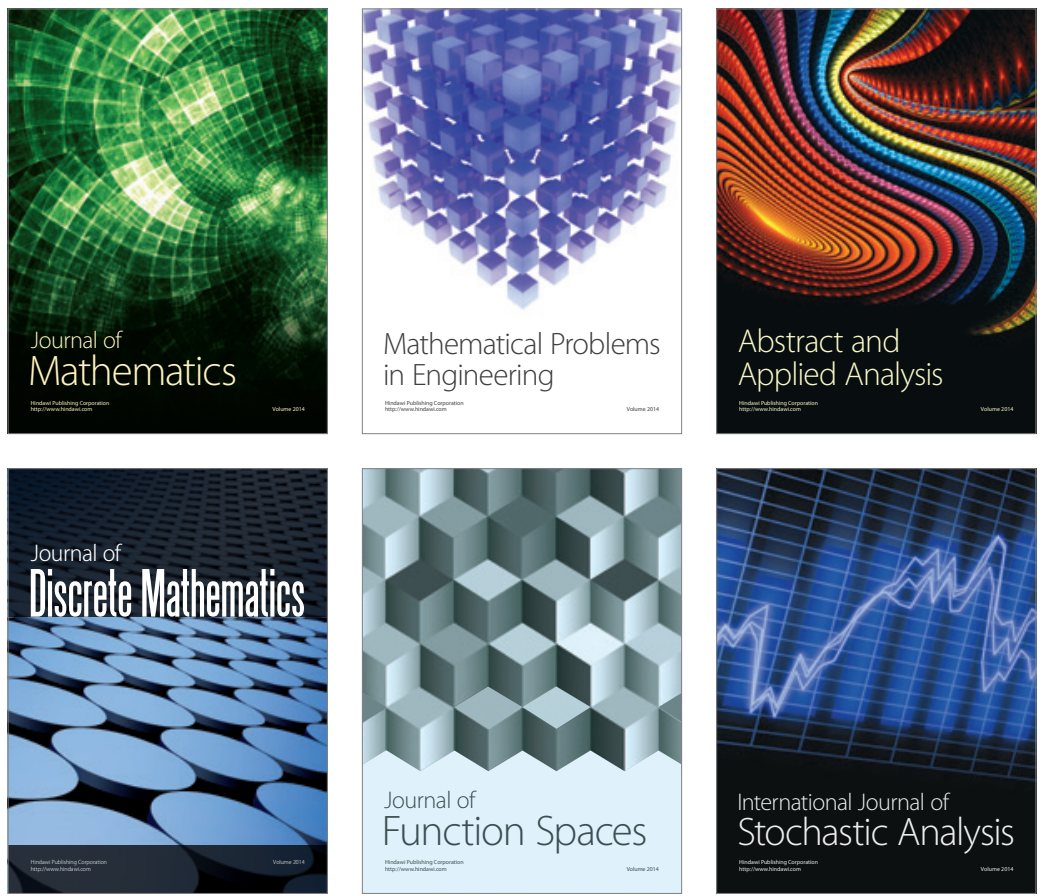

Journal of

Function Spaces

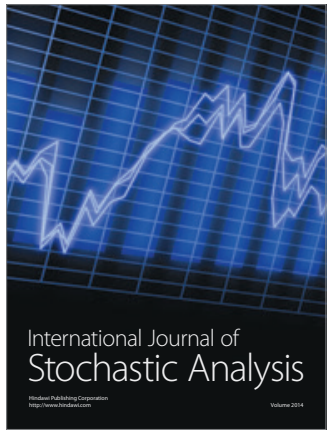

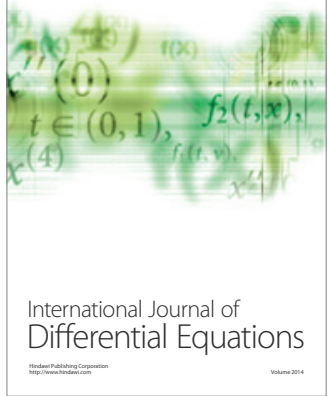
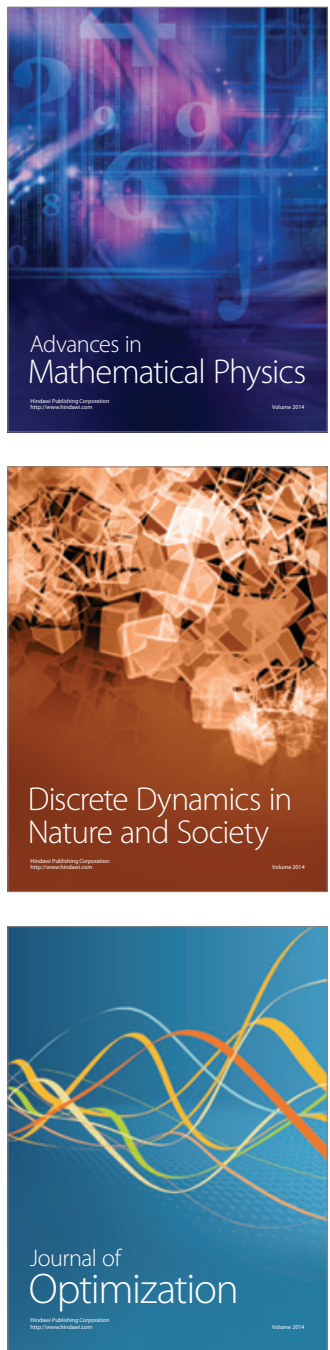